\title{
AN EXPERIMENTAL ANALYSIS ON EFFECT OF NOISY IMAGE DATASETS ON PERFORMANCE OF DEEP LEARNING MODELS
}

\author{
Modalavalasa Hari Krishna1, Dr.Makkena Madhavi Latha ${ }^{2}$ \\ ${ }^{1}$ Research Scholar, Dept.of ECE, JNTUH-CEH, Telangana, India. \\ ${ }^{2}$ Professor, Dept.of ECE, JNTUH-CEH, Telangana, India.
}

\begin{abstract}
Deep Learning is a sub field of Machine Learning which works based on neural network structures. Deep Learning has been increasing the capabilities of Artificial Intelligent systems rapidly in many emerging areas like video analytics, data analytics and autonomous systems. The availability of the labelled data is increased due to enhancements in digital technology. The generation and labelling of training data become easier as many advanced and low-cost sensors are developed. Even though various sensors are available, Noise is the one of the major concerns of the database creation. In case of limited availability of data, Noise can be used to normalize the model to avoid overfit. But in huge databases, noise decreases the inference accuracy of the trained model. So, it is very important to understand the effect of noise on model performance. This paper presents an experimental analysis on the individual and combined effect of noise present in training database and validation database. Here, Standard CIFAR-10 database with 60000 images is used as labelled data. A comparative analysis is made to observe the behavior of trained model in terms of inference accuracy against three types of injected noises such as Gaussian Noise, Salt \& Pepper Noise and Speckle Noise at different variance levels.
\end{abstract}

Key Words: Deep Learning, Inference Accuracy, Model Overfit, Noise effect, Training Accuracy, Validation Accuracy.

\section{INTRODUCTION}

Nowadays Artificial Intelligent (AI) or machine intelligence is very emerging technology which is intelligence demonstrated by machines, in contrast to the natural intelligence displayed by humans. AI has its applications in most of scientific, computational, financial, marketing and entertainment sectors to reduce the human effort, human errors and processing time. Autonomous vehicles, Data Mining, Big Data, Robotics and video analytics are few real time applications of AI [2][3]. Machine Learning (ML) is sub-field of AI, which deal with autonomous part of AI application. Many ML algorithms are developed to support many real time applications in both supervised and unsupervised ML models. Neural Networks are one of best ML algorithms, which reduce the human efforts further by extracting the features by itself. In general, these Neural Networks are known as Deep
Learning (DL) models as they look deep into data to extract the meaningful features for classification or regression with many hidden layers between input layer and output layer [4][5].

Artificial Neural Networks (ANN), Recurrent Neural Network (RNN) and Convolutional Neural Networks are well known examples of Deep Neural networks [6][7][8]. The major difference between an ANN and CNN is that only the last layer of a CNN is fully connected whereas in traditional ANN, each neuron is connected to every other neuron. RNN is highly applicable for temporal data or sequential data. CNN has higher feature compatibility than RNN and more powerful than RNN [9].

Training and validation are two important phases in deep learning model creation. Most of the times these two processes repeated for many iterations to adjust model weights in order get maximum accuracy. Usually these DL models require huge amount of training and validation datasets to attain desired accuracies. Small datasets may overfit the model and effect validation or inference accuracies. Addition of noise into training dataset may restrict the overfit but requires more effort to converge the model [1]. In large datasets overfitting is not considerable problem but noise present in the training or validation datasets badly effect the model accuracy and may leads to model divergence. So, one should understand the effect of different noises present in the training dataset or validation dataset to generate the convergent model with desired accuracy. Gaussian, Speckle and Salt \& Pepper noise models are commonly used noise environments to simulate different real time electrical and mechanical noises added during image database creation and transmission [10][11]. So, in this paper, these 3 noise models are considered at different variance levels and injected into standard image database to determine the model behaviour with noisy database.

\section{METHODOLOGY}

In this work, we have considered 4-layer deep neural network model (CNN) with 3 hidden layers. Each hidden layer has one Convolutional layer, one Batch normalization layer and one ReLu layer. Size of input layer is $32 \times 32 \times 3$ and output layer size is 10 . The considered CIFAR-10 data set has 60000 color (Standard RGB color) Images with size $32 \times 32$. All these 60000 images are collected from 10 
different classes [12]. Out of these 60000 images, 50000 images are used for training and 10000 images used for validation. This training is limited to 5 epochs to reduce the training time as the dataset size is huge. To determine the effect of noise on network model accuracy, 3 different noise models have been considered such as Gaussian noise, speckle noise and Salt\& Pepper noise. These noise models are injected into all the CIFAR-10 dataset images at different noise variance levels such as $0.01,0.05,0.1,0.3,0.5,0.8$.

Here we have considered 4 different cases in order to determine the individual and combined effect of noise present in training datasets and validation datasets. In case-1, noise is injected into both training datasets and validation datasets to observe the combined noise effect on model accuracy. In case-2, noise is added to only training image dataset and trained model is validated with original CIFAR-10 validation dataset to observe the individual effect of noise present in training data. In case-3, model is trained with actual CIFAR-10 train dataset without any noise injection and validated using CIFAR-10 validation dataset with added noise to observe the individual effect of noise present in it. In case-4, actual CIFAR-10 training and validation datasets trained and validated without any noise injection.

\section{Results \& Discussions}

The 4-layer deep learning model is designed, trained and validated in MATLAB r2019b software. Simulation setup uses a workstation laptop with advanced Hexa-Core Intel Core-i7 [ 9th generation- 9750] processor with Nvidia GTX 1660-Ti GPU and 32GB RAM. All model training and validation processes directed to GPU cores to reduce the training durations. First the model is trained and validated with original CIFAR-10 datasets without any noise injections (case-4) and epochs and iterations are adjusted to attain accuracy more than $60 \%$. Considered 4-layer Deep learning model attained nearly $62 \%$ accuracy after 5 epochs and 2000 iterations. After that, remaining 3 cases (case-1,2\&3) are simulated one by one. Each case (except

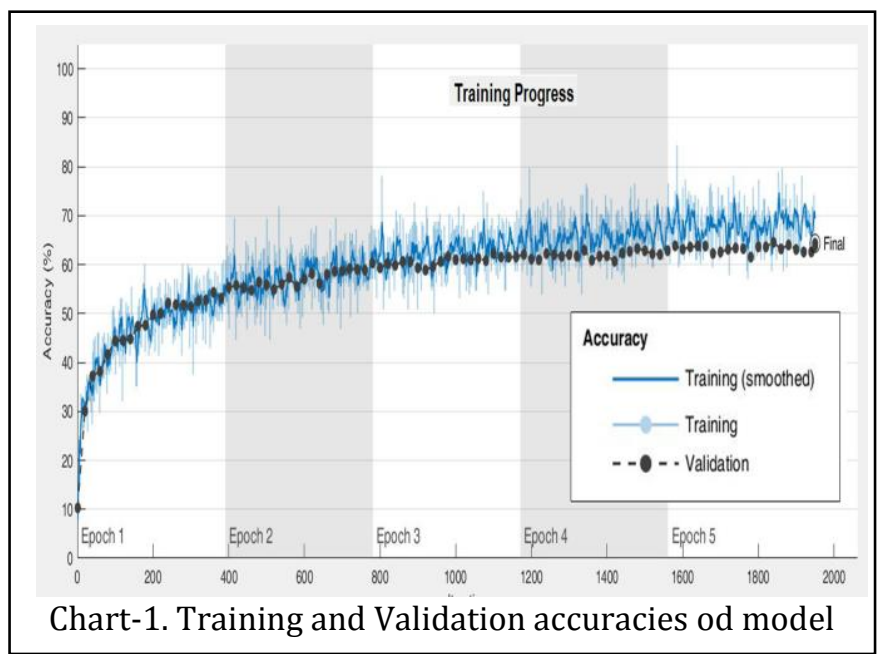

case-4) is simulated in 18 different noise scenarios (each of three considered noise models at 6 variance levels, $3 \times 6=18$ ). So, considered model is trained and validated for 55 times using datasets with different noise models at different variance levels and all results are consolidated to determine the deep learning model behavior in noise environment.

The training and validation are performed in parallel manner at each iteration and training accuracy reached to $50 \%$ within first epoch. The training and validation plots of case-4 are shown in Chart-1. X-axis represents the model accuracy and iteration count is taken on Y-axis. The Blue color lines indicate training accuracy and black color lines indicate validation accuracy. In case-4, as both training and validation image datasets are noise-free, the accuracy levels are increased with same improvement rate. This observation also valid for case- 1 but in case- 2 , training dataset is noise free and validation dataset is noisy, so validation accuracy fell down below the training accuracy line as shown in Chart-2. Here, the interesting thing is, even though case- 2 and case- 3 are opposite in noise nature, their models are not exhibiting the behaviors in same manner. In case- 3 , the model behavior is almost stable with very little fluctuations, irrespective of injected noise levels or noise models.

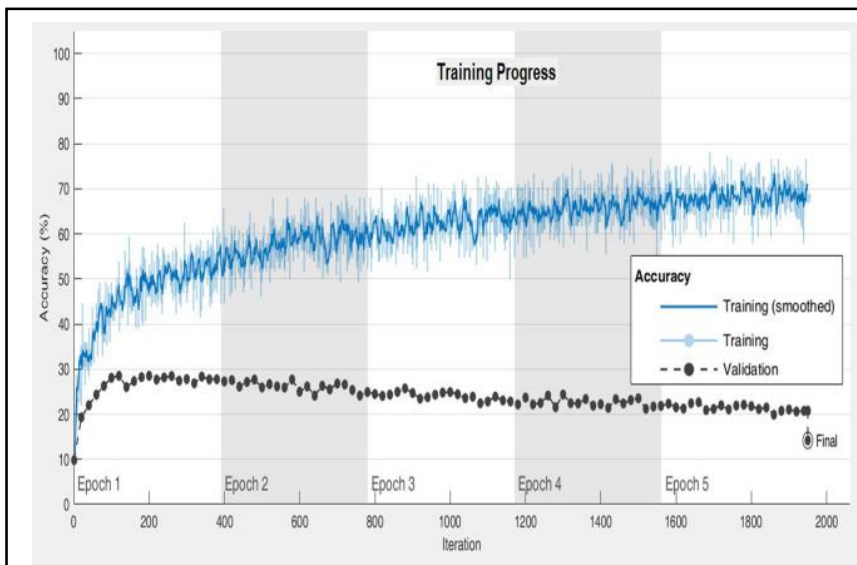

Chart-2. Training and Validation accuracies od model in case-2

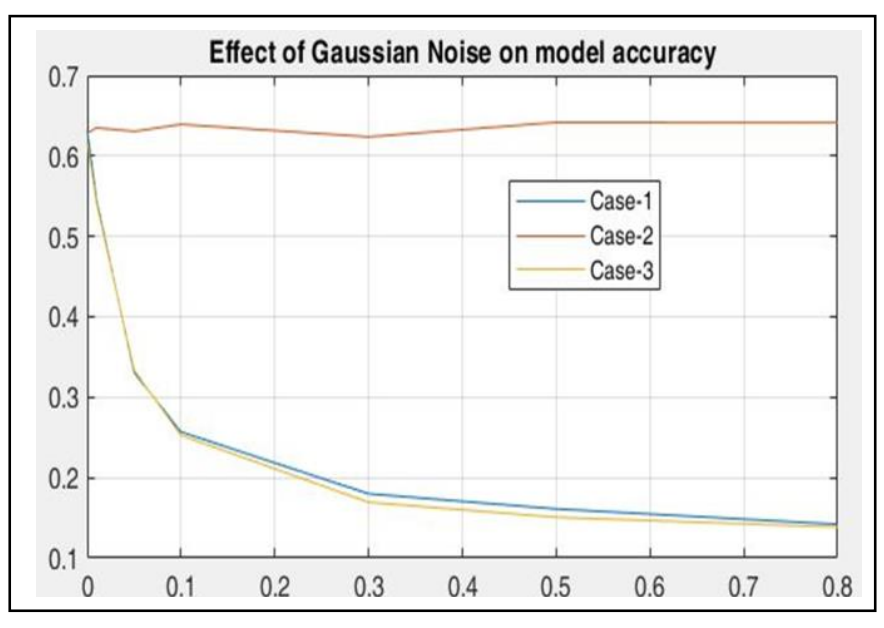


Chart-3 shows the effect of gaussian noise on model accuracy (Validation accuracy levels). Here variance levels are taken on X-axis and accuracy levels are taken on Yaxis. In Gaussian noise environment, case- 1 and case- 3 are showing same behavior but case-2 is showing stable model accuracy. Chart-4 shows the model behavior in speckle noise environment and Chart-5 shows the model behavior in Salt \& Pepper noise environment. Here, one can observe that all considered noise models are showing
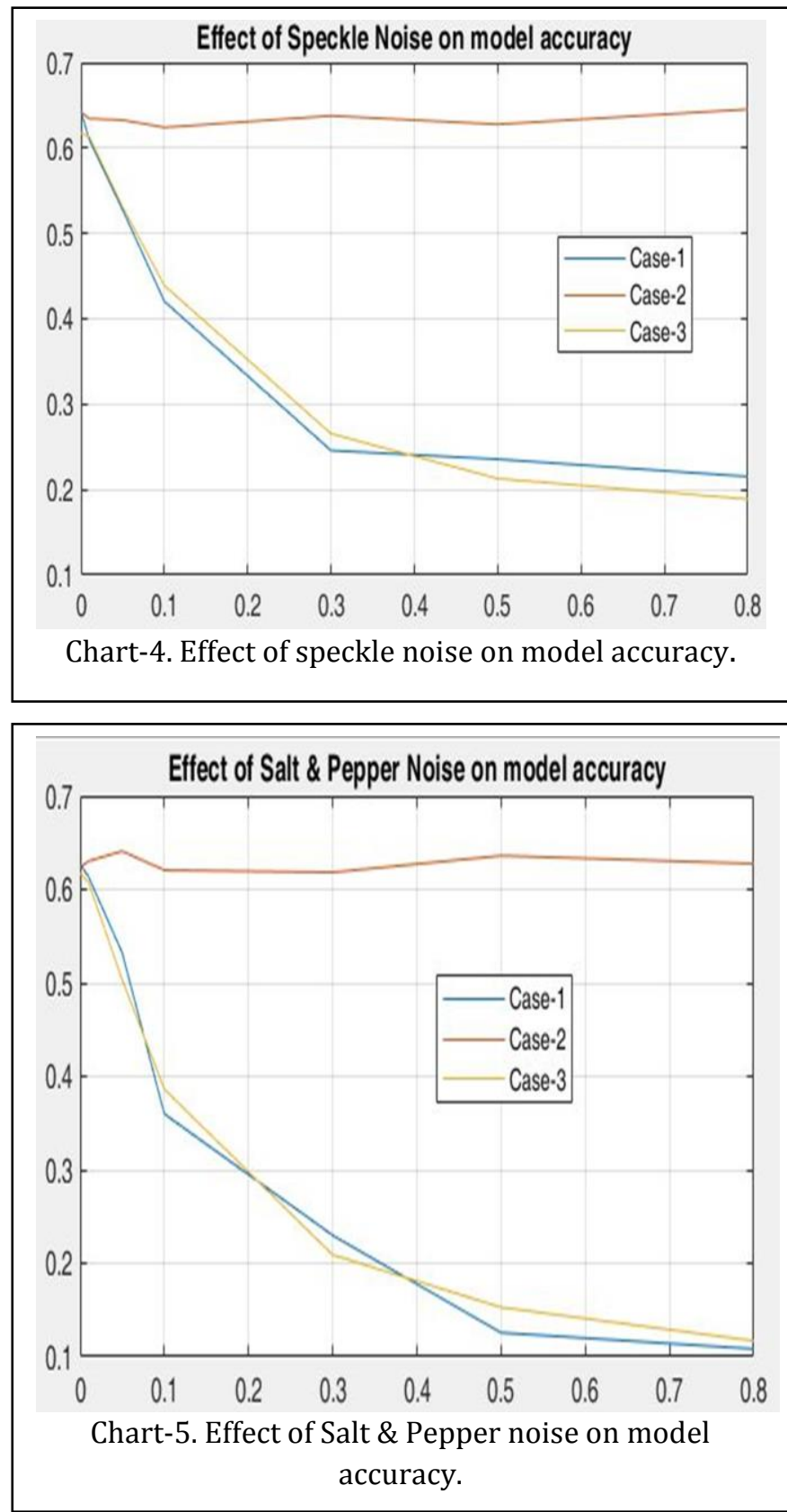

almost same behavior. After noise model wise analysis, the results are analyzed case wise, in case- 1 and case- 3 the model behaves in same manner in all noise environments and model validation behaviors of case- 1 and case- 3 are shows in Chart- 6 and Chart-7 respectively. But the case- 2 exhibited completely different behavior with nearly stable
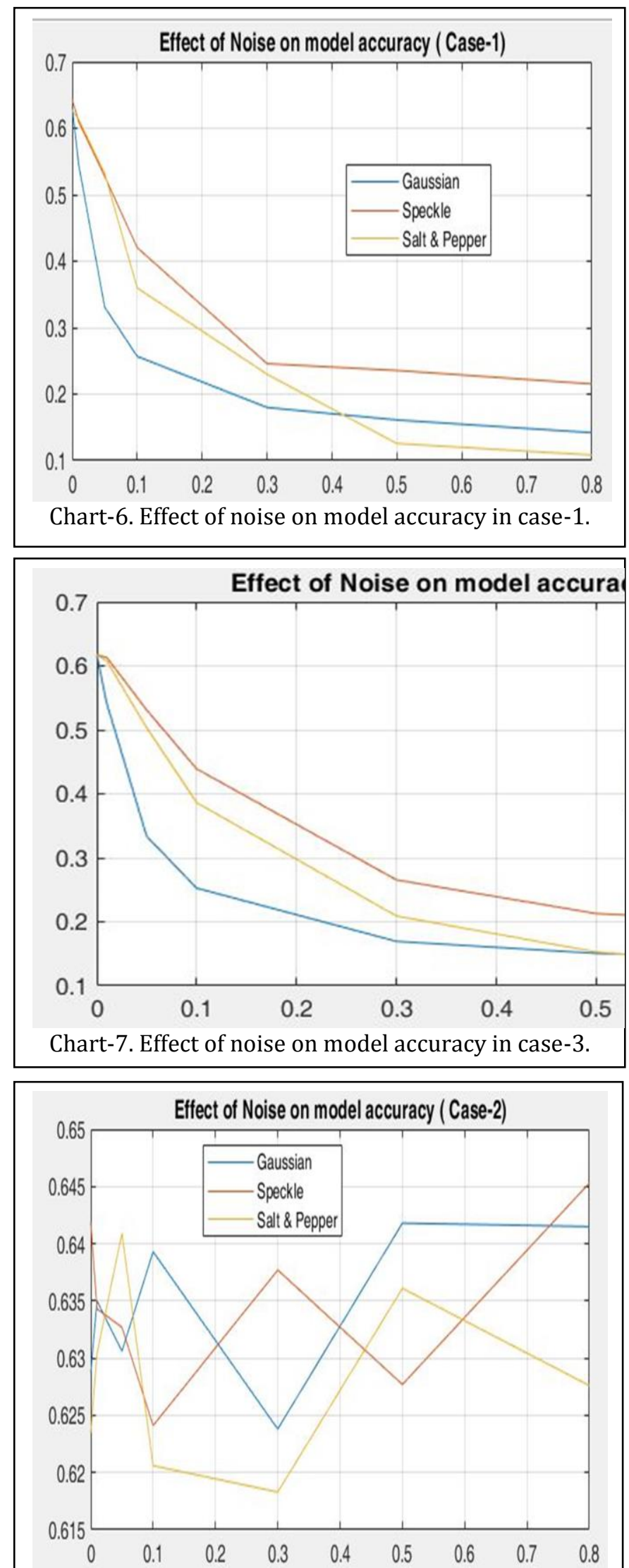

Chart-8. Effect of noise on model accuracy in case-2. 
validation accuracy in all 19 scenarios ( 3 noise models $\times 6$ noise levels +1 noise free scenario) as shown in Chart-8. All the results from simulated 55 scenarios are consolidated and plotted in Chart-9. The case- 2 results are completely on different manner, whereas case-1 and case2 results are following same way, that is, with increase in noise levels, model accuracy is decaying rapidly (nearly exponential decay). With clear observation, it is evident that, Gaussian noise mostly effects the model accuracy when comparing with other two, whereas speckle noise effect on model accuracy is least at all variance levels. The effect of Salt \& Pepper noise is low at lower noise levels and higher at higher noise levels than Gaussian and
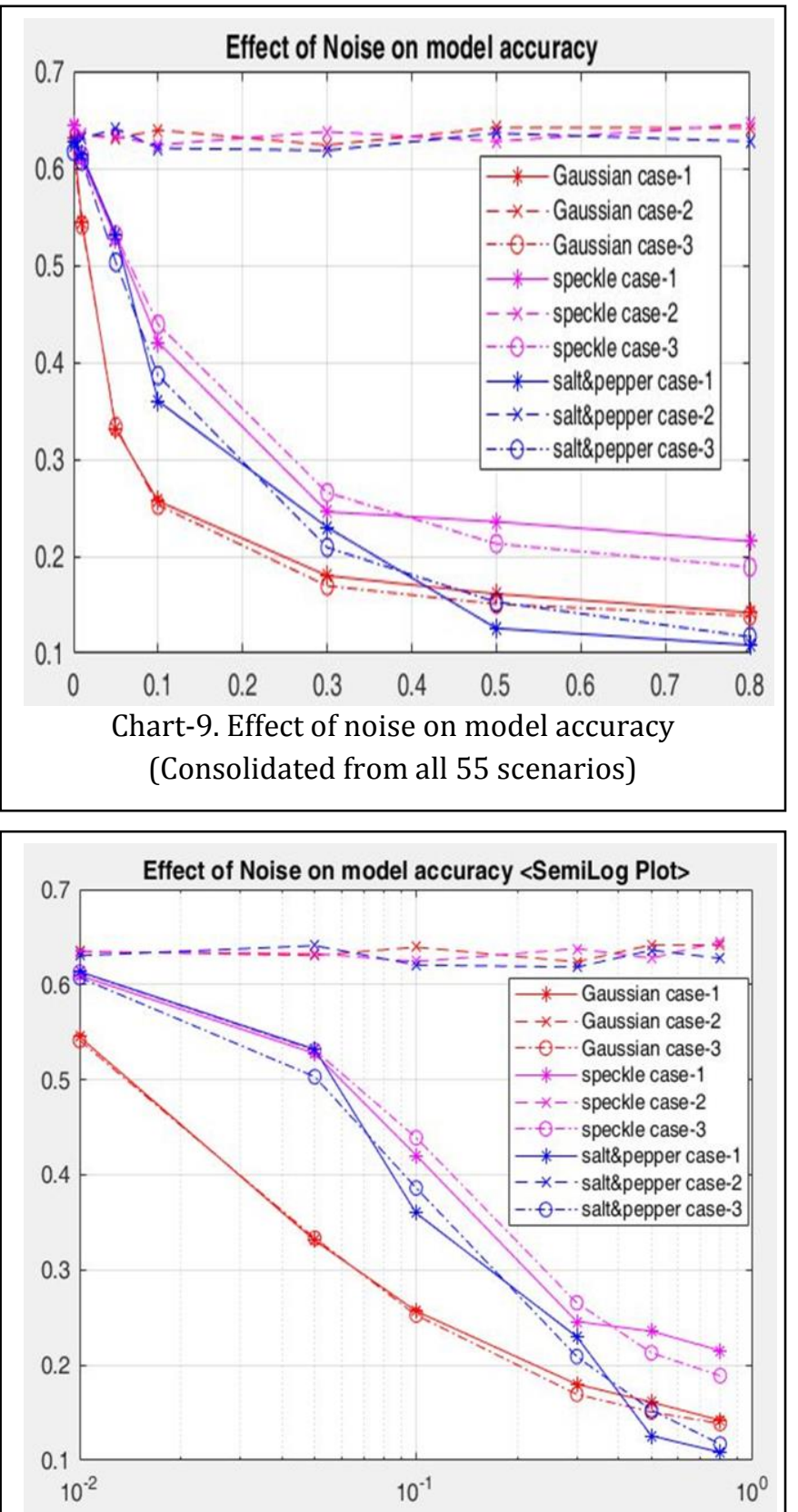

Chart-10. Effect of noise on model accuracy in semilog plot (Consolidated from all 55 scenarios)

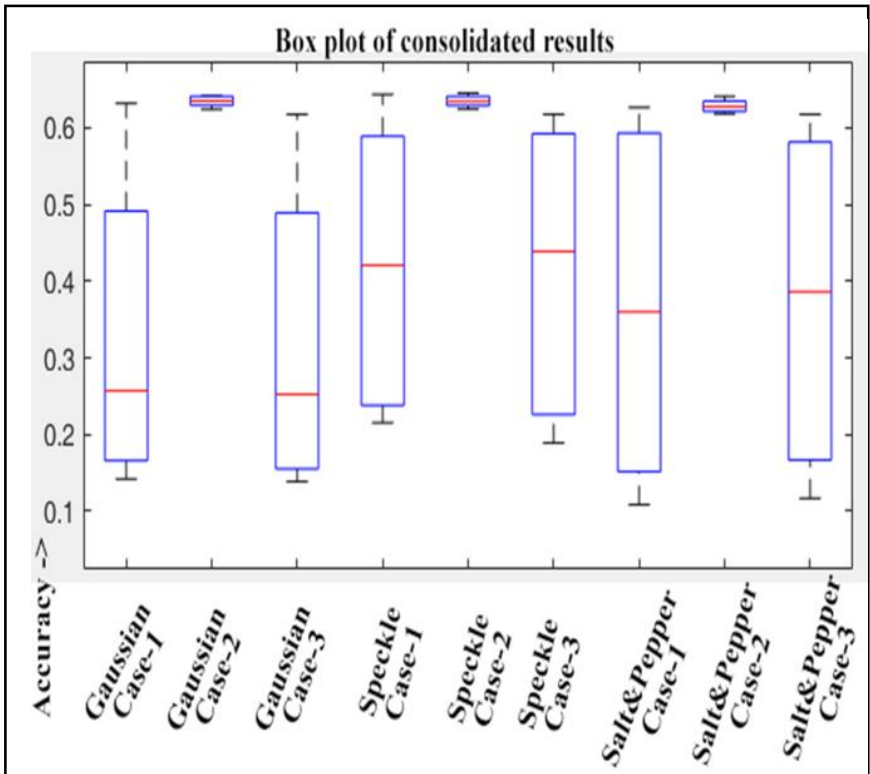

Chart-11. Effect of noise on model accuracy in box plot (Consolidated from all 55 scenarios)

Speckle noises.

These consolidated results are plotted on semi-log graph as shown in Chart-10 to represents the behavior of deep learning model at lower variance levels. The Gaussian and Salt \& Pepper noises effects the model very badly at higher noise levels and make it divergent with model accuracies less than 10\%. These results from all simulated 55 scenarios state that, case- 1 and case- 3 are similar and exhibiting decaying accuracies with increased noise levels, and case- 2 exhibiting stable accuracies irrespective of noise levels. We can synthesize these results and state that model accuracy mainly depends on validation dataset or inference dataset only. The individual effect of noise present in training dataset is very less compared to effect of noise present in validation or inference dataset. This noise injection into training dataset (case-2) also increased the model accuracy up to $2 \%$ as shown in Chart11. This increase in model accuracy is due to restriction of model overfit by added noise.

\section{CONCLUSIONS}

The effect of noise present in image datasets on deep learning models is experimentally simulated using a 4layer deep learning model. Gaussian, speckle and Salt \& Pepper noise models are added to standard CIFAR-10 dataset at different variance levels and designed deep learning model is trained and validated in 55 different scenarios. The individual and combined effect of noise present in training dataset and validation dataset are analyzed in 4 different cases. Among the considered three noise models, Gaussian noise mostly effecting the model 
accuracy and speckle noise is least effecting one whereas effect of Salt \& Pepper noise very high at higher noise levels that other two models. The model may get diverge with validation data with high amount of noise content as the validation accuracy falls below 10\%. With clear observation of consolidated results, it is evident that, the deep learning network model accuracy is decaying rapidly with increased noise level in validation dataset irrespective of noise level present in training dataset. The individual noise present in training dataset improves the model accuracy by decreasing the model overfit during training.

\section{REFERENCES}

[1] Saseendran, Arun \& Setia, Lovish \& Chhabria, Viren \& Chakraborty, Debrup \& Barman Roy, Aneek, "Impact of Noise in Dataset on Machine Learning Algorithms", 2019,10.13140/RG.2.2.25669.91369.

[2] Li Deng; Dong Yu, "Deep Learning: Methods and Applications," in Deep Learning: Methods and Applications , now, 2014.

[3] Sampo Kuutti; Saber Fallah; Richard Bowden; Phil Barber; Amir Khajepour, in "Deep Learning for Autonomous Vehicle Control: Algorithms, State-of-theArt, and Future Prospects,", Morgan \& Claypool, 2019.

[4] A. S. Modi, "Review Article on Deep Learning Approaches," 2018 Second International Conference on Intelligent Computing and Control Systems (ICICCS), Madurai, India, 2018, pp. 1635-1639, doi: 10.1109/ICCONS.2018.8663057.

[5] Fu-Lian Yin, Xing-Yi Pan, Xiao-Wei Liu and Hui-Xin Liu, "Deep neural network language model research and application overview," 2015 12th International Computer Conference on Wavelet Active Media Technology and Information Processing (ICCWAMTIP), Chengdu, 2015, pp. 55-60, doi: 10.1109/ICCWAMTIP.2015.7493906.

[6] H. Yanagisawa, T. Yamashita and H. Watanabe, "A study on object detection method from manga images using CNN," 2018 International Workshop on Advanced Image Technology (IWAIT), Chiang Mai, 2018, pp. 1-4, doi: 10.1109/IWAIT.2018.8369633.

[7] J. Chen and H. Zhuge, "Extractive Text-Image Summarization Using Multi-Modal RNN," 2018 14th International Conference on Semantics, Knowledge and Grids (SKG), Guangzhou, China, 2018, pp. 245248, doi: 10.1109/SKG.2018.00033.

[8] G. Margi and B. Talati, "Automated medical image segmentation using RBF ANN," 2017 International Conference on Information, Communication, Instrumentation and Control (ICICIC), Indore, 2017, pp. 1-5, doi: 10.1109/ICOMICON.2017.8279052.

[9] Y. Sun, B. Liu and X. Xu, "An OpenCL-Based Hybrid CNN-RNN Inference Accelerator On FPGA," 2019 International Conference on Field-Programmable Technology (ICFPT), Tianjin, China, 2019, pp. 283286, doi: 10.1109/ICFPT47387.2019.00048.

[10] H. Chen, "A kind of effective method of removing compound noise in image," 2016 9th International Congress on Image and Signal Processing, BioMedical
Engineering and Informatics (CISP-BMEI), Datong, 2016, pp. 157-161, doi: 10.1109/CISPBMEI.2016.7852700.

[11] M. Y. Jabarulla and H. Lee, "Evaluating the effect of various speckle reduction filters on ultrasound liver cancer images," 2018 International Conference on Electronics, Information, and Communication (ICEIC), Honolulu, HI, 2018, pp. 1-4, doi: 10.23919/ELINFOCOM.2018.8330656.

[12] R. Doon, T. Kumar Rawat and S. Gautam, "Cifar-10 Classification using Deep Convolutional Neural Network," 2018 IEEE Punecon, Pune, India, 2018, pp. 1-5, doi: 10.1109/PUNECON.2018.8745428.

\section{BIOGRAPHIES}

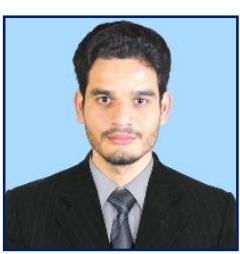

Mr. Modalavalasa Hari Krishna is currently pursuing his full-time Ph.D from Jawaharlal Nehru Technological University-Hyderabad (JNTU-H). He had completed Post-Graduation in VLSI System Design and awarded with GoldMedal. He completed B.Tech in Electronics and Communication Engineering(ECE). He had published one international journal and one International Conference paper. He had attended many National level conferences and Workshops. His research areas of interest are Machine Learning, Deep Learning, Image Processing, Automation and VLSI design. He has 4 years of real time Industrial experience. The author may be reached at mhkrishna9s@gmail.com.

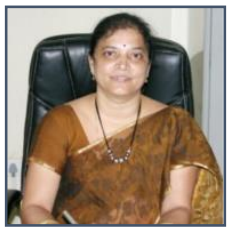

Dr. Makkena Madhavi Latha is currently working as Professor in Electronics and Communication Engineering Department, JNTUH-CEH and Director, Academic \& Planning, JNTU-Hyderabad. She completed her Ph.D from JNT University - Hyderabad in 2002. She has 25 years of experience in teaching and 3 years of experience in real time Industry. She worked as Director, Innovative Technologies, JNTUH. She also has 18 years of experience in research. She handled 09 Research and Development projects and established 6 new laboratories. She published more than 180 research papers in National/ International Journals and Conferences and 2 patents. She was awarded with many national and International awards along with State Best Teacher award from Govt. of Andhra Pradesh. She is a life member of ISTE and Member of IEEE. She guided 25 Ph.D scholars, 228 M.Tech students and 103 B.Tech projects. She organized and coordinated many international and national level conferences, seminars and workshops. 